\title{
Simulation capabilities and application results of the Geant4 Toolkit
}

\author{
Maria Grazia Pia* $\dagger$ \\ INFN Sezione di Genova, 16146 Genova, Italy \\ E-mail: Maria.Grazia.Pia@cern.ch'
}

ABSTRACT: Geant4 is a toolkit for the simulation of the passage of particles through matter, developed according to advanced software engineering techniques and methodologies. Its applications cover a variety of experimental domains: high energy and nuclear physics, astrophysics, space science, bio-medical physics and radiation background studies.

\section{Introduction}

Geant4 [i]1] [i] through matter:

- geometry and materials

- particle interaction in matter

- tracking management

- digitisation and hit management

- event and track management

- visualisation and visualisation framework

- user interface

The Geant4 software was developed by scientists. Since 1999 the production service, user support and development of Geant4 have been managed by the international Geant4 Collaboration.

The first Geant4 production version was published in December 1998. Since then new versions have been regularly released, extending and improving the functionalities of the Toolkit. The Geant4 source code is freely available from iGeant 4 web sitê, accompanied by an extensive set of documentation, examples and training material.

${ }^{*}$ Speaker.

${ }^{\dagger}$ On behalf of the Geant4 Collaboration 


\section{The role of software engineering in Geant4}

Software engineering plays a fundamental role in Geant4. Advanced software engineering techniques are adopted in Geant4 in response to the requirements of functionality, modularity, extensibility and openness.

The life-cycle model adopted for most domains in Geant 4 is both iterative and incremental. The steps among Analysis of Requirements, Design, Implementation and Testing are repeated. Refinements and extensions to the design are applied according to new requirements or performance issues.

Problem domain and use-case analysis led to definition of the User Requirements [2i] during the initial phase of the project. User Requirements have been systematically reviewed and updated following the ESA PSS-05 software engineering standard [i $\left[\begin{array}{l}\overline{4} \\ 1\end{array}\right]$.

The Booch [3i] methodology has been employed for the Object-Oriented Analysis and Design. Problem domain decomposition and Object Oriented Analysis and Design have led to a clear hierarchical structure of sub-domains, linked by a uni-directional flow of dependencies. The UML notation is adopted as the common language for the documentation of designs. OOAD is supported by the use of the Rational Rose CASE tool [i్ji]

The Geant 4 Object Oriented design allows the user to understand, customise or extend the toolkit in all the domains. At the same time, the modular architecture of Geant4 allows the user to load and use only the components needed.

The development of effective tests and testing procedures has been a major effort in Geant4, as part of the software process. It involves a dedicated Testing and Quality Assurance working group and various classes of testing, such as unit and integration or system testing. As with the other software methodologies, it has been guided by established and well-documented practices in software engineering. Although unit and system testing are critical to ensuring the integrity and correctness of the Geant4 code, it is also important to build quality in from the start. To this various Quality Assurance tools are employed, consisting of both commercial products as well as internal coding guidelines.

In order to achieve maintainable software and ensure its quality, the adoption of standards, wherever possible, is promoted. Encapsulation of components is maximised, interdependency and unit complexity is minimised.

A plan for Software Process Improvement has been adopted by the Geant4 Collab-

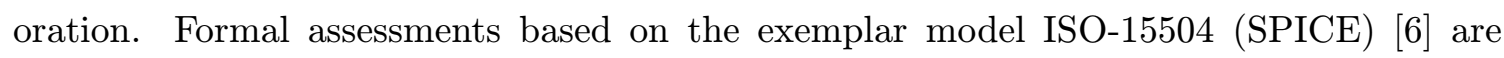
performed. SPI is considered life-cycle driven, therefore progresses of the established program are constantly monitored.

\section{Overview of Geant4 main functionalities}

The Geant4 kernel handles the management of runs, events and tracking. The Event package provides an abstract interface to external physics event generators for the creation of the primary particles. Advanced functionalities are provided to perform sophisticated studies, suc as, for instance, the simulation of pile-up effects. 
Geant4 Tracking handles the propagation of a track, determined by physics interactions. It is completely general and common to all processes for any particle types, thus allowing a great flexibility in the implementation of a variety of physics processes, as well as the openness to further extensions. In order to fully exploit the validity ranges of the physics models, Geant4 does not apply any tracking cuts, but relies only on production thresholds. In addition, Geant 4 can ensure a consistent and material-independent accuracy of the simulation, as the production cuts are set in terms of particle range.

The management of particles is based on Particle Data Group compliant definitions and data, including their decay processes and modes.

The role of Geometry in Geant 4 consists in providing the user the ability to describe the detailed geometrical structure of a detector; it also handles the equation of motion solvers in different fields and geometrical boundaries conditions for the propagation of particles. Geant4 Geometry provides an ISO STEP compliant solid modeller, allowing the exchange of models from CAD systems. Multiple solid representations, such as Constructive Solid Geometry or Boundary Represented Solids (including NonUniformRationalBSplines) are available. Boolean operations on solids are supported.

The Geant4 Materials package allows to describe materials consisting of a single element or a mixture of elements, which in turn can consist of a single isotope or a mixture of isotopes. The physical properties of materials can be described in a generic way by quantities which can be either given directly, like density, or derived from the element composition.

The Hits and Digi domains provide the functionality to reproduce the read-out structure of the detector and its electronic response, independently from the geometry used for the tracking.

A fast parameterisation facility is integrated with the full simulation, allowing independent and simplified detector descriptions and direct production of hits. The fast simulation can be triggered according to various options, such as particle type, volume etc.

Geant4 Visualisation provides the capability to visualise detector geometry, particle trajectories, tracking steps, hits, texts (character strings) etc. Its design, based on object oriented abstract interfaces, makes Geant4 independent from any particular graphics system; at the same time it allows multiple implementations of drivers to interface the simulation with a variety of graphical systems. A similar approach if adopted for the User Interface domain, allowing the usage of a variety of user interfaces, from simple commandline driven ones to sophisticated GUIs.

Extensive possibilities of interaction with the Geant4 system are offered to the user via a set of dedicated user-action classes.

A wide set of utilities, including physics units and constants, and interface to ODBMS complete the Geant4 Toolkit.

\section{Geant4 physics: design and capabilities}

The Geant4 Toolkit provides extensive physics capabilities, through an abundant set of physics processes, that make it suitable for applications in an ample variety of domains. 
One of the most important goals of Geant4 consists in making the design and implementation of the physics open and transparent, thus contributing to the validation of experimental results. The Geant4 design makes physics data, models and assumptions transparently accessible to the user - rather than hard-coded in black-box packages, thus improving the verification and the reliability of simulation results. It also exposes the granular implementation of the physics, each component of which can be inspected at source code level. Thanks to the object oriented tecnology, a variety of alternative or complementary physics models can be provided for the same physical process, with openness to further extensions.

The way cross sections are calculated - via formulae, parameterisations or interpolation of databases - is interchangeable. The information extracted from the database is separated from the way it is accessed and used, giving the opportunity of using different databases and allowing their applicability to be tailored by particle, energy, material, etc. Similarly the generation of the final state is separated from the calculation of the cross-sections used for tracking and is also available as alternative or complementary models, according to the energy, range, the particle type and material.

Whenever available, use is made of the public evaluated databases distributed by a variety of international sources; this contributes to the reliability and the openness of the physics implementation.

Geant4 electromagnetic physics [8i] provides a variety of implementations of electron, positron, photon and charged hadron interactions. Photon processes include Compton and Rayleigh scattering, $\gamma$-conversion and photoelectric effect. Electron/positron processes handle bremsstrahlung, ionisation and $\delta$-ray production, positron annihilation and synchrotron radiation. The ionisation and energy loss of hadrons and ions is handled as well. The multiple scattering process can handle all charged particles, computing the mean path length correction and the mean lateral displacement. Low energy extensions - down to 250

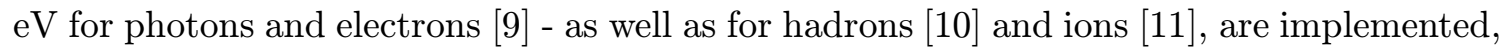
with multiple models. The validity range of all the muon processes, based on theoretical models, scales up to the thousand $\mathrm{PeV}$ region, allowing the simulation of ultra-high energy and cosmic ray physics.

Geant4 can also handle the optics of scintillation and Cerenkov detectors and their associated light guides, including a set of dedicated processes for optical photons: refraction and reflection at medium boundaries, bulk absorption and Rayleigh scattering.

The Geant4 Toolkit provides an ample set of physics models for the simulation of hadronic interactions. The models follow three different basic approaches: data-driven, parameterisation-driven and theory-driven modelling; in the overall framework Geant4 offers both complementary and alternative models. The hadronic models all follow the basic approach adopted throughout Geant4, that consists in exposing the physics to the users through the object oriented design, thus contributing to the transparency required for the validation of experimental results. 


\section{Conclusions}

Geant4 represents one of the largest and most ambitious projects in the domain of software for high energy physics experiments, in terms of the size and scope of the code and the number of contributing scientists. It has demonstrated that rigorous software engineering practices and object-oriented methods can be profitably applied to the production of a coherent and maintainable software product, even with the fast-changing and open-ended requirements presented by physics research.

Geant 4 has been adopted by several experiments, space science and medical physics projects as the basis for their simulations. Such a wide domain of applications offers also the opportunity for technology transfer [i] ini] from high energy physics to other fields. A large set of application results [133] are available at the time of writing these Proceedings, including also comparisons between experimental data and Geant4-based simulations.

\section{References}

[1] RD44 Collaboration, Status Report, CERN/LHCC-98-44, 1998.

[2] RD44 Collaboration, User Requirements Document, 1998.

[3] G. Booch, Object-Oriented Analysis and Design with Applications, The Benjamin Cummings Publishing, 1994.

[4] ESA, Guide to User Requirements Definition Phase, ESA PSS-05, 1994.

[5] I. White and M. Goldberg, Using the Booch Method, a Rational Approach, The Benjamin Cummings Publishing, 1995.

[6] ISO/IEC Joint Technical Committee 1 (JTC1), ISO/IEC DTR 15504-5, 2000.

[7] D.E. Groom et al., 'Eur. Phys. J.

[8] S. Chauvie et al., Proc. of the Monte Carlo 2000 Conference, Springer, 2000.

[9] Apostolakis J et al., INFN/AE-99/18, 1999.

[10] Giani S et al., INFN/AE-99/20, 1999.

[11] Giani S et al., INFN-AE-99/21, 1999.

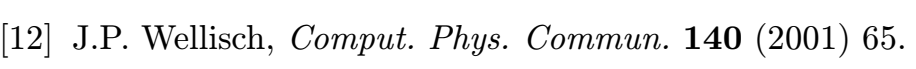

[13] J. Apostolakis et al., Proceedings of CHEP 2001.

[14] S. Chauvie et al., Proceedings of CHEP 2001 (2001) 41. 